\title{
Nanoética, un nuevo campo para viejos problemas del riesgo tecnológico
}

\author{
Nanoethics, a new field for old technological risk \\ problems
}

\author{
JORGE ENRIQUE LINARES SALGADO \\ Facultad de Filosofía y Letras, UNAM (México)
}

Recibido: 15-10-2012

Aprobado definitivamente: 17-11-2012

\section{RESUMEN}

Las nanotecnologías constituyen actualmente el sector más innovador y desafiante en el desarrollo tecnocientífico-industrial. Dado que nuestras tecnociencias no son capaces de crear sistemas que sean tanto o más consistentes, más estables y autosustentables que los sistemas naturales, son necesarias e indispensables la supervisión y la regulación prudencial y precautoria de sus efectos y consecuencias, esperadas e inesperadas, mediante un debate público con amplia participación democrática. La nanoética es una forma de poner en acción una racionalidad colectiva prudencial que, en vistas del alcance omniabarcador de las nanotecnologías, tiene que ser construida a partir de principios éticos de alcance global y debe tener un carácter prospectivo.

PALABRAS CLAVE

NANOTECNOLOGÍA, REGULACIÓN, RIESGO TECNOLÓGICO, NANOÉTICA

\section{ABSTRACT}

Nowadays nanotechnologies constitute the most innovative and challenging sector in technoscientific-industrial development. Our technosciences aren't capable of generating equally or more consistent stable and self-sufficient systems than natural systems. Therefore, supervision together with reasonable and precautionary regulation of their effects and consequences -being these expected or unexpected-, in the form of public debate with ample democratic participation 
proves to be necessary. Nanoethics is a way of starting a reasonable collective rationality movement which, in view of the all-containing span of nanotechnologies, must be constructed from ethic principles that have a global reach and must as well have a prospective character.

KEYWORDS

NANOTECHNOLOGY, REGULATION, TECHNOLOGICAL RISK, NANOETHICS

\section{LeIBNIZ PLANTEÓ EN SU Monadología (1715):}

65. [...] cada parte de la materia no sólo es divisible al infinito, como reconocieron los antiguos, sino que está de hecho subdividida sin fin en otras partes, cada una de las cuales tiene un movimiento propio [...]

66. De donde es evidente que en la más mínima parte de la materia hay un mundo de criaturas, de vivientes, animales, entelequias, almas.

67. Cada parte de la materia puede ser concebida como un jardín lleno de plantas y como un estanque lleno de peces. Pero cada rama de la planta, cada miembro del animal, cada gota de sus humores es también como ese jardín o ese estanque.

68. Y aunque la tierra y el aire, que hay entre las plantas del jardín, o el agua, que hay entre los peces del estanque, no son ni planta ni pez, contienen, sin embargo, otras plantas y otros peces, tan sutiles, empero, casi siempre, que no podemos percibirlos.

69. Así, no hay nada inculto, estéril y muerto en el universo; el caos y la confusión son sólo aparentes; como si se mira un estanque a cierta distancia, desde la cual se vislumbra un movimiento confuso y, por decirlo así, un revoltijo de peces, sin llegar a discernir los peces mismos.

A partir de esas intuiciones leibnicianas sobre la complejidad estructural de la materia podemos advertir que las nanotecnologías (cuyo propósito central es producir materiales con nuevas propiedades y funciones, como resultado de intervenciones moleculares y atómicas en la materia inerte y en organismos vivos), constituyen actualmente el sector más innovador y desafiante en el desarrollo tecnocientífico-industrial. Entre las propiedades que se busca potenciar están la reactividad, la conductividad y la mayor resistencia y ligereza de los 
materiales, además de la producción de nanopartículas, nanotubos, fulerenos, nanocables, nanosensores y sus combinaciones en nanoartefactos cuya aplicabilidad es multifuncional y se extiende a muy diversos campos como la medicina, la remediación del medio ambiente, los materiales sintéticos, la energía y las comunicaciones electrónicas.

Más allá de las enormes expectativas, pero también de las exageraciones mediáticas que han suscitado las nanotecnologías, algunos de sus proyectos a mediano y largo plazo (principalmente en nanobiotecnología y en la producción de nanomáquinas) podrían no sólo transformar la materia de un modo radical, sino manufacturarla y (re)diseñarla (de arriba abajo o de abajo arriba), combinando de una manera inédita lo orgánico y lo inorgánico, lo artificial y lo natural: la materia física y la materia biológica.

Las nanotecnologías conforman así una plataforma tecnológica sin precedentes no sólo por la convergencia interdisciplinaria, ya que integran la química, la física, la biología molecular, la ciencia de los materiales, la optoelectrónica, la informática y las ciencias cognitivas, entre otras, sino por la amplitud de los proyectos tecnocientíficos, de cuyos resultados se esperan aplicaciones para la manufacturación de productos nanotecnológicos en todos los sectores industriales.

Ahora bien, la tesis principal de la que parto es la siguiente: dado que nuestras tecnociencias no son capaces de crear sistemas que sean tanto o más consistentes, más estables y autosustentables que los sistemas naturales, son necesarias e indispensables la supervisión y la regulación prudencial y precautoria de sus efectos y consecuencias, esperadas e inesperadas, mediante un debate público con amplia participación democrática.

El debate ético sobre las nanotecnociencias debe responder a los mismos cuestionamientos que dirigimos a otras innovaciones: ¿Cuáles son los beneficios ostensibles, las necesidades reales a que responden, y cómo se distribuyen y a qué costo? ¿Cuáles son los riesgos y los efectos imprevisibles para la salud, para el medio ambiente, para la equidad socioeconómica; en qué medida son controlables? ¿Quién y cómo puede y debe regular las producciones nanotecnológicas? ¿Quiénes son los principales beneficiados, en el caso en el que se produzcan beneficios?

Por consiguiente, todas las nanopartículas y nanoartefactos, así como los métodos y sistemas para su fabricación deben ser analizados, regulados y tratados como entidades y procesos novedosos, pues no tienen precedentes; ello 
es congruente con el hecho de que sean patentables, y no como exigen ciertos sectores de la industria, como en el caso de los OGM: no regularlos aplicando el doble rasero moral basado en el falaz principio de «equivalencia sustancial». ${ }^{1}$

Por tanto, los problemas éticos que suscitan las nanotecnociencias son los mismos que los de otras ramas tecnológicas convencionales: riesgos ambientales, sanitarios y sociales, así como el problema mundial de la desigualdad e inequidad en la distribución y acceso a sus beneficios. Pero en lo particular, las nanotecnociencias agudizan también estos problemas:

1. Privatización del conocimiento.

2. Brecha socio-económica y cognitiva Norte-Sur.

3. Secretismo y opacidad industrial de espaldas a la sociedad.

4. Brecha de artificialidad (en sistemas bio-artificiales que no son equivalentes a los organismos naturales generados por la evolución).

Asimismo, las nanotecnociencias han reabierto cuestiones filosóficas de fondo: por ejemplo, ¿hasta dónde podemos manipular las estructuras de la materia y de la vida, y controlar esos efectos?; y sobre todo, ¿cuáles son las consecuencias de la interacción o incluso fusión de moléculas orgánicas con las inorgánicas? Por ello, el problema del riesgo tecnológico emerge directamente con nuevas implicaciones con los nano-artefactos que ya se diseñan y operan, puesto que en la escala nano se producen interacciones desconocidas e imprevisibles entre lo orgánico y lo inorgánico. De este modo, las nanotecnociencias implican una nueva dimensión del riesgo tecnológico, ya que:

1. No hay posibilidad de un control técnico completo, como ocurriría con las tecnologías macromoleculares y macroscópicas, pues el nivel de incertidumbre a nivel atómico y molecular es mucho mayor.

2. Las cualidades y propiedades nanométricas abren posibilidades asombrosas pero también nuevas responsabilidades en cuanto a los riesgos y extrernalidades negativas; tres propiedades son objeto primario de una nueva responsabilidad ética: invisibilidad, indeterminación, autoreplicación (en el caso de la bio-nanopartículas).

3. Los marcos políticos y legales de la tecnociencia actual son peligrosamente insuficientes y vagos para regular a las nanotecnociencias; dejan demasiada iniciativa a las industrias y refuerzan la cultura imprudente y las políticas anti-precautorias que predominan en go-

1 La estrategia de comercialización mundial de transgénicos se ha basado en patentes que protegen las «originalidad» y novedad de estos bioartefactos; pero la industria se ha empeñado en evitar toda regulación especial alegando que los transgénicos son «sustancialmente» equivalentes a otros organismos naturales, es decir, no intervenidos por la biotecnología. 
biernos y en los actores industriales del mercado. Las nanociencias están subordinadas a los intereses de la concentración del capital y de las ganancias comerciales a corto plazo, además del apoyo estratégico de los Estados en proyectos de orden militar-bélico.

Ante estos nuevos (en realidad viejos) riesgos tecnológicos, ha surgido en respuesta una nueva modalidad de ética aplicada: la nanoética, que está emparentada con otras éticas aplicadas que ya se han consolidado, como la bioética o la ética ambiental. La nanoética es necesariamente interdisciplinaria e inevitablemente prospectiva: tiene que anticiparse mediante el uso de experimentos mentales y de analogías históricas, experiencias previas y teorías prospectivas, a lo que efectivamente será posible en el futuro. Aquí conviene señalar que la nanoética no debe dar por buena sin más cualquier proyección, deseo o temor, aunque sea generalizado, con respecto a los proyectos nanotecnológicos; ni darlos por realidades inexorables anticipadamente mediante una especie de determinismo tecnológico. Lo que debe tomar en cuenta para hacer sus prospectivas son los conocimientos validados, las tendencias de desarrollo del mercado capitalista mundial, los contextos sociales, políticos y culturales, así como las consecuencias ontológicas (y todavía incipientes) de lo que implicaría esta nueva fase del desarrollo técnico-industrial.

Las nanotecnologías constituyen el modelo más acabado de lo que varios filósofos y científicos sociales hemos denominado tecnociencia. Ésta es una nueva modalidad, históricamente desarrollada desde la segunda mitad del siglo pasado, para generar conocimiento, a partir de esquemas conceptuales reduccionsitas, y dirigidos directamente a producir innovaciones tecnológicoindustriales de interés comercial y estratégico. La tecnociencia, a diferencia de la ciencia teorética y de las ramas tecnológicas convencionales, se despliega en proyectos inter y multidisciplinarios, reticulares y distribuidos en varias partes del mundo, mediante el financiamiento de grandes inversiones de empresas y gobiernos; ${ }^{2}$ por ello, es, a la vez, el motor fundamental de la acumulación del capital en la economía global y de la innovación tecnológica. Los proyectos nanotecnológicos poseen carácter estratégico para las empresas y los gobiernos, y ambos confluyen en algunos proyectos con claros fines militares. La tecnociencia concentra el poder tanto epistémico, técnico, comercial, como político y militar.

Esta alta concentración del poder en pocas manos, regida por la dinámica del mercado capitalista mundial, contribuye a acrecentar la desigualdad e inequidad socioeconómica. Y estas consecuencias sociales que crean mayor

2 La Iniciativa Nacional en Nanotecnología de los EE.UU. ha destinado 5000 millones de dólares sólo en el periodo 2001-2005. Un tercio de este presupuesto corresponde a proyectos de finalidad militar. 
malestar social y político no pueden ser ignoradas por los agentes impulsores del cambio tecnológico, ni por los científicos y tecnólogos que actualmente trabajan en dichos proyectos.

Otra de las consecuencias de enorme trascendencia filosófica que ya despunta en los logros actuales de las nanotecnologías es un nuevo campo de hibridación tecnológica, lo cual conlleva también formas distintas de interacción sociedad-naturaleza y nuevas dinámicas sociales en el mundo globalizado, además de la movilización del imaginario colectivo mediante representaciones mediáticas, mitos, temores y esperanzas colectivos.

La tesis que propongo ahora requiere mayores precisiones, pero lo formularé en términos generales: las nanotecnociencias, en sus diversas dimensiones y modalidades, generarán mediante su conjunción un ámbito insospechado de nuevos objetos artefactuales, es decir, de entidades artificiales: nuevos híbridos mitad naturales, mitad artificiales; así como diversas y contradictorias representaciones sociales y estéticas. En particular, una nueva modalidad de bioartefactos son los que llaman la atención y suscitan asombro, pero también preocupación por los efectos desconocidos de la interacción entre la materia inerte y la materia orgánica a niveles moleculares y atómicos, en donde la incertidumbre es regla y no excepción.

Durante siglos, los seres humanos pudieron distinguir claramente entre las cosas hechas por la naturaleza y las cosas hechas por la técnica (esta es la distinción clásica de Aristóteles en su Física, libro II). No obstante, desde que comenzamos a domesticar animales y plantas (hace miles de años) también se inició, de hecho, la primera hibridación natural-artificial para dar lugar a lo que ahora podemos denominar genéricamente bioartefactos: organismos vivos domesticados, alterados, modificados e intervenidos en diversos niveles técnicos de complejidad y control, desarrollados a lo largo de la historia técnica. Hemos podido, por tanto, modificar y dominar a diversos organismos vivos alterando su conducta, su fisiología, su estructura genética o su composición molecular, para responder así eficazmente a finalidades técnicas que los seres humanos asignamos de manera extrínseca (artificial) a las funciones naturales propias (evolutivas) de esos organismos. Asimismo, la técnica humana se ha concentrado desde antaño en la replicación, sustitución o simulación artefactual de entidades, procesos, órganos o sistemas naturales. Llamaremos a este tipo de artefactos, de acuerdo con la tipología de Negrotti, naturoides. ${ }^{3}$ Pues bien, sea por la artificialización de algunos componentes de organismos vivos para convertirlos en bioartefactos, sea por el intento de imitar a la naturaleza (biomímesis) mediante naturoides, los artefactos que hemos diseñado y construido nunca constituyeron una nueva clase de entidades, distintas a las que describió Aristóteles (o bien

3 Vid. Massimo Negrotti, Naturoids, 
seguían siendo organismos naturales, o bien artefactos artificiales fabricados mecánicamente con materia inerte). Pero a partir del surgimiento de las nanotecnologías, en particular la bionanotecnología, se abre la posibilidad de lograr una hibridación estructural sin precedentes que daría por resultado una categoría ontológica distinta a las entidades que conocemos en el mundo. Se trata, pues, de una hibridación molecular y/o atómica entre lo natural y lo artificial, entre lo orgánico y lo inorgánico, acelerada y potente, que no requerirá los cientos o miles de años de adaptación y coevolución, como sucedió con los primeros bioartefactos surgidos de la domesticación tradicional.

Estas hibridaciones serán distintas e inéditas porque justamente las propiedades físicas y biológicas que se busca generar serían novedosas y también indeterminables, hasta cierto punto. Pero estos bio-nano-artefactos, aunque se basan en los modelos, bases estructurales y funcionales de los sistemas orgánicos, replican mecanismos artefactuales simples (nuestras máquinas tradicionales de circuitos causales cerrados) y reducen la complejidad de las interacciones entre los seres vivos (y entre éstos y el ambiente) a unas cuantas acciones y efectos mecánicos, que se espera controlar de algún modo, mediante esquemas pragmáticos.

Recurro otra vez a las agudas observaciones de Leibniz. Siguiendo la clásica distinción aristotélica entre las cosas naturales y las cosas hechas por la técnica, Leibniz establece este postulado:

64. Así en cada cuerpo orgánico de un viviente hay una suerte de máquina divina o un autómata natural que sobrepasa a todos los autómatas artificiales. Porque una máquina hecha por el arte humano no es máquina en todas sus partes. Por ejemplo, el diente de una rueda de metal tiene partes o fragmentos que no son ya, para nosotros, nada artificial ni poseen nada que tenga carácter de máquina con respecto al uso a que la rueda está destinada.

Pero las máquinas de la naturaleza, o sea, los cuerpos vivos, son máquinas hasta en sus más mínimas partes, hasta el infinito. Esta es la diferencia entre la naturaleza y el arte; es decir, entre el arte divino y el humano.

65. Y el Autor de la naturaleza ha podido hacer este artificio divino e infinitamente maravilloso porque cada parte de la materia no sólo es divisible al infinito, como lo han reconocido los antiguos, sino que está actualmente subdividida sin fin en otras partes, cada una de las cuales tiene un movimiento propio, que de otro modo sería imposible que cada porción de la materia pudiera expresar el universo todo.

He ahí la fuente de la tesis general que he planteado al inicio: dado que nuestras tecnociencias no son capaces de crear sistemas que sean más consistentes y 
sustentables que los sistemas naturales, es previsible que la artificialidad de lo que produzca (así sea a partir de organismos y materia vivos) implique resultados y consecuencias inesperados, algunos de ellos negativos y muy riesgosos, por lo que será necesaria una supervisión y una regulación social precautoria, mediante un proceso de participación ciudadana amplia y democrática. El viejo modelo de desarrollo tecno-industrial tiene que dar paso a un nuevo modelo más atemperado y que responda a los intereses más amplios de la sociedad.

Pues bien, podemos plantear, al menos como hipótesis, que las nanotecnociencias están en la antesala de una transformación material que hará más complejas nuestras «máquinas» (ahora miniaturizadas), si es que llega a ser posible lograr que adquieran las propiedades que Leibniz señaló: imitar, reproducir o replicar las máquinas biológicas; o bien en sentido contrario: artificializar las micro máquinas biológicas al convertirlas en micro-bioartefactos. De este modo, tendremos una nueva dimensión nano de bioartefactos y naturoides. Habremos dejado atrás el período milenario de domesticación de plantas y animales para dar lugar a una nueva era de bio-manufactura industrializada. Esta transformación tecno-económica implicará, dicho sea de paso, una serie de profundas transformaciones socioculturales, económicas, estético-simbólicas, ético-políticas.

Desde una perspectiva filosófica, es posible postular desde ahora, en esta etapa todavía incipiente en resultados artefactuales (aunque ya se utilizan más de 600 productos nanofacturados), que en su interacción sistémica, los diversos proyectos nanotecnocientíficos producirán una segunda hibridación ontológica en la historia, que daría lugar a nuevos tipos de entidades en el mundo:

a) artefactos u objetos técnicos que funcionen como si fueran objetos orgánicos (con capacidades como autoreplicación o de adaptación y coevolución);

b) objetos técnicos cada vez más orgánicos o construidos con materiales orgánicos; y asimismo,

c) objetos naturales (organismos vivos) reconfigurados o intervenidos molecularmente (bioartefactos), aunque sólo fuera por la acción de nanopartículas en algunos de sus tejidos o células.

Este cambio técnico puede parecer que se reduce a un pequeño salto cuantitativo, pero si sumamos lo que conocemos y lo que desconocemos del comportamiento de la materia a niveles nano, podemos suponer que esta capacidad técnica ampliada tendrá efectos imprevisibles, y algunos de ellos de un alcance verdaderamente ontológico. 
Por ello, hay que considerar seriamente dicho alcance potencial, pero con la prudencia debida, para no validar cualquier temor o confianza excesiva. Por tanto, la nanoética es una forma de poner en acción una racionalidad colectiva prudencial que, en vistas del alcance omniabarcador de las nanotecnologías, tiene que ser construida a partir de principios éticos de alcance global. Las nanotecnologías podrían homogeneizar formas de la materia, y esto es en sí mismo un riesgo en un mundo natural que es, por excelencia, diverso, sobre todo en el mundo de la vida. Pero mucho antes de que tal uniformidad de la materia se hiciera realidad (aunque sea en una menor medida), debemos enfrentar un efecto uniformador que ya genera diversos problemas, tanto simbólicos como materiales: las nanotecnociencias se extenderán, muy probablemente, sin ninguna limitación ni regulación social efectiva, traspasando fronteras, regulaciones nacionales, códigos legales, así como sus nanopartículas pueden traspasar sin problemas los cuerpos de personas, animales y plantas. Si la nanotecnología es, por primera vez, una tecnociencia que nace globalizada; es decir, cuyo potencial de expansión es claramente universal, a diferencia de otras tecnologías, como las informáticas, que dependen no sólo de la distribución de mercancías (artefactos electrónicos) sino también de conocimientos y cultura de uso, las nanopartículas no tendrían mayor obstáculo para expandirse que la distancia geográfica.

Pero ello implica otro problema esencial: la nanoética debe tener un carácter prospectivo, debe anticiparse a la realización de esos proyectos nanotecnológicos y no sólo enfrentar los problemas presentes o ya verificados. Por eso, el mundo de las nanotecnociencias constituye un nuevo campo de discusión y de práctica de principios éticos prospectivos, que ya se han aplicado a otros campos tecnocientíficos.

Considero que los principios ético-políticos con los que surge la nanoética deben ser análogamente de potencial alcance universal. Los principios generales que he propuesto en otras investigaciones como mínimos para activar el debate social sobre las tecnociencias son estos cuatro: la responsabilidad colectiva, la precaución, la protección de la autonomía de personas y de comunidades, y finalmente, la justicia distributiva.

Así pues, la nanoética debe acompañar el avance de las nanotecnologías en todo el orbe. Tenemos que aprovechar la oportunidad histórica de lograr un modelo de desarrollo más equilibrado y regulado socialmente, para beneficio de todos, incluyendo en nuestras consideraciones prudenciales a otros seres vivos a los que podemos dañar o afectar irreversiblemente. El enorme desafío que tiene en sus manos la nanoética consiste, por ende, en impulsar políticas que promuevan y protejan la equidad y el acceso público a los beneficios, la seguridad ambiental, la bioseguridad humana y de otros seres vivos, la priva- 
cidad y la autonomía informacional, la interacción regulada y controlada entre dispositivos artificiales y organismos vivos.

He propuesto en otro texto ${ }^{4}$ la formación de un sistema de principios éticopolíticos que orienten las discusiones públicas y las decisiones políticas sobre el desarrollo tecnológico. Dichos principios éticos no sólo tienen por objetivo reducir los riesgos y evitar la posibilidad de catástrofes tecnológicas de gran alcance e impacto, sino también potenciar equitativa y globalmente los beneficios que cualquier tecnología sea capaz de producir.

Particularmente controversial y problemático ha resultado la aplicación del principio de precaución. Si se buscan nuevas propiedades de la materia con modificaciones nanométricas, entonces es razonable esperar que algunas de estas propiedades inéditas conlleven efectos inesperados e imprevistos. El riesgo aumenta en la medida en que crece la incertidumbre sobre algún efecto causal en la naturaleza. La nanoética tendrá que navegar inevitablemente en el mar tempestuoso del principio de precaución.

Las principales críticas al principio de precaución se han dirigido a lo costoso que resulta y, sobre todo, a que en realidad no es posible predeterminar los eventos futuros, por lo que parecería en ocasiones una medida excesiva y que detendría irracionalmente el desarrollo científico y tecnológico. Sin embargo, aun en las aplicaciones más radicales del principio de precaución (como las moratorias) no hay motivo para decir que detienen u obstaculizan la investigación científica. Por el contrario. Lo que obliga la aplicación correcta y razonada del principio de precaución es a revisar y monitorear los artefactos producidos, asî como sus efectos sobre el ambiente y la salud de muchos seres vivos. El principio de precaución tendría tres niveles de aplicación en la nanotecnología:

1. Riesgos de interacciones estructurales y macrosistémicos, difíciles de prever y/o de controlar.

2. Riesgos específicos de toxicidad en organismos vivos y de efectos inesperados de las nanopartículas en el ambiente.

3. Riesgos de invasión de la privacidad y pérdida de autonomía en algunos desarrollos nanoinformáticos.

Ahora bien, el núcleo mismo de la responsabilidad social consiste en una regla primordial: someter toda innovación tecnocientífica a una evaluación previa de los fines, medios y sus posibles efectos sobre la naturaleza y sobre la sociedad. Pero no solamente es preciso evaluar sus efectos evidentes, in-

4 Vid. J.E. Linares, Ética y mundo tecnológico, 2008. 
mediatos y ostensibles, sino también sus probables efectos, sus consecuencias latentes y no inmediatas. Debemos monitorear y supervisar cada tecnología cuidadosamente y nunca cerrar por completo otras alternativas; mas sólo será legítimo desactivar o retirar un sistema tecnológico si poseemos evidencias de riesgos de envergadura mayor. Cuando existe la posibilidad de riesgos mayores, el principio de responsabilidad se transforma en el principio de precaución: vale más la prudencia o incluso la abstención de la acción que incurrir en un riesgo mayor. Pero además nos obliga a modificar, controlar o eliminar toda tecnología que tenga un poder destructivo incontrolable e irreversible sobre la naturaleza o las condiciones de vida humana, como lo son los armamentos nucleares, químicos o biológicos, o como podrían ser algunos experimentos en nanotecnología. En este nivel, debemos proceder analizando y monitoreando una a una las innovaciones nanotecnológicas.

Es necesario evaluar y supervisar cualquier innovación nanotecnocientífica, con estudios científico-técnicos, sociales y ambientales sin conflicto de interés y mediante un debate abierto y plural. La conciencia de la responsabilidad tecnocientífica depende de la adecuada difusión pública de las investigaciones sobre los riesgos y las consecuencias de las tecnologías. Estas investigaciones pueden extenderse para que dispongamos así del tiempo suficiente para ponderar en un debate público razonado estas consecuencias y para poder asumir los riesgos que sean necesarios o inevitables, pero contando con el consentimiento informado de las personas y de las comunidades. Por ello, el principio de responsabilidad nos obliga a desacelerar el desarrollo tecnocientífico, a extender el tiempo de las pesquisas y las inspecciones, así como el de los debates públicos, siempre que sea factible, pero de ningún modo implica coartar ni prohibir el desarrollo tecnocientífico.

Así pues, un factor decisivo para evaluar imparcialmente los riesgos tecnológicos y garantizar una adecuada responsabilidad social es la investigación científica independiente de los intereses industriales y comerciales, que esté al servicio de los Estados y de organismos internacionales para realizar una adecuada evaluación del riesgo, para inspeccionar y supervisar las innovaciones y buscar mejoras a las tecnologías existentes. Asimismo, dicha investigación independiente tiene como fin la difusión más amplia posible de información corroborada, la educación científica de la sociedad y la consolidación de una cultura pública tecnocientífica.

El carácter complejo de los nuevos nano-bio-artefactos que se introducen en el mundo implica que debe ponerse límites a algunos intereses que corresponden sólo a determinados sectores sociales, como los que están centrados únicamente en el lucro o rentabilidad económicos (aunque se revistan de una retórica humanitarista). Aquí puede haber conflictos con las libertades económicas y las necesidades productivas estratégicas de las naciones. Por eso la regla 
fundamental derivada de la responsabilidad social nos indica que los proyectos tecnocientíficos deben ser sometidos, desde sus primeras fases, al escrutinio público y al debate entre diferentes agentes sociales para que se garantice la seguridad y un nivel de riesgo aceptable, en función de intereses legitimados y consensuados (la salud, la bioseguridad, la protección ambiental, la equidad social, el desarrollo sustentable, la justicia socioeconómica, la protección de las diferencias culturales, los derechos de las minorías étnicas, etc.).

Análogamente a la nanotecnología, la nanoética debe ser construida de un modo estructural distinto al de otras éticas aplicadas contemporáneas, justamente para responder a los nuevos problemas que están planteando las producciones nanotecnológicas. Desde este punto de vista, la nanoética tendría que ser un campo de reflexión ética interdisciplinaria (nanociencias y filosofía) para prever, anticipar, establecer precauciones y orientar el desarrollo justo y sustentable de esa revolución tecnocientífica en marcha. La oportunidad histórica (pero también el desafío) son únicos: por primera vez una revolución tecnocientífica podría ser adecuadamente acompañada de una reflexión y debate público rigurosos, mediante procedimientos democráticos para lograr un mejor modelo de desarrollo, sustentable, seguro, equitativo. De lo contrario, si la nanotecnología sólo es guiada por los factores e intereses de corto plazo que dominan en el mercado capitalista mundial, y por los sistemas de decisión tecnocráticos, a espaldas de los ciudadanos y sus necesidades reales, los riesgos y desigualdades sociales que puede provocar serán inevitables, y se combinarán peligrosamente con los problemas ya creados por otras tecnociencias.

Si hemos dicho que la nanoética tendrá que ser conformada de una manera distinta a otras éticas aplicadas, de manera interdisciplinaria, prospectiva y prudencial, en redes de investigación global que tengan acceso directo a los proyectos en marcha, ello implica también una nueva arquitectura, una estructura reticular de sus principios y de sus configuración social.

Desde la Declaración de Budapest de 1999 venimos hablando de un «nuevo contrato social» para la ciencia y la tecnología. Ello conlleva la necesidad de realizar modificaciones en las formas de participación ciudadana para poder tomar decisiones con mayor base de racionalidad y legitimidad. Esta participación ciudadana es indispensable para asegurar el adecuado control y orientación de la tecnociencia en la reducción de sus riesgos inherentes y la potenciación de sus beneficios. Los ciudadanos deben involucrarse mediante diversos mecanismos y formas de representación porque ellos son los evaluadores y destinatarios finales de los sistemas tecnológicos, y porque debe reconocerse, además, que las valoraciones morales y los intereses son plurales en las sociedades actuales y, por eso, ninguno de ellos posee el privilegio de contar con verdades y conocimientos incuestionables. Así pues, debemos dejar atrás el modelo tradicional de desarrollo técnico-industrial en el que sólo intervenían los científicos y tecnólogos, o sólo 
los políticos asesorados por los primeros, y los demás ciudadanos quedaban al margen como receptores y usuarios pasivos.

Así pues, la consolidación de una nanoética, como de otras éticas aplicadas a los problemas sociales y ambientales de las tecnociencias, requiere que las sociedades innoven sistemas de participación ciudadana democrática para evaluar y supervisar los sistemas tecnológicos en vistas de reducir los riesgos y daños, así como de ampliar de manera equitativa sus beneficios materiales. Ello implica que los ciudadanos estén mejor informados y que quienes participen en las deliberaciones también estén dispuestos a aprender, cambiar sus opiniones, cuestionar sus prejuicios, a escuchar a los expertos y a todos los involucrados, a estar abiertos a todas las posiciones y consideraciones, para no descartarlas sin más o por influencia de sus propios prejuicios e intereses. Las controversias tecnocientíficas pueden constituirse como un medio privilegiado para fortalecer una cultura democrática deliberativa, una forma colectiva de phrónesis que eduque a todos los ciudadanos (legos y expertos, gobierno y sociedad civil) y que armonice, en la medida de lo posible, los distintos intereses políticos, económicos, ambientales con base en criterios ético-políticos de mayor consenso para todas las comunidades.

Será complicado lograr un equilibrio entre la precaución y la innovación; pero es posible alcanzar un modelo mucho mejor de desarrollo. Para ello, la primera y más indispensable exigencia a las nanotecnociencias y nanoindustrias es la transparencia de la información, el monitoreo y la trazabilidad de todos los productos. En segundo términos, regulaciones estrictas de seguridad («nanoseguridad») para los experimentos controlados y, sobre todo, para la producción industrial y su liberación comercial; así como regulación de la publicidad comercial para evitar falsas expectativas. Tercero, procedimientos eficientes y acordados globalmente para la evaluación sistémica de riesgos sociales y ambientales. En estos procedimientos es indispensable establecer formas innovadoras y eficaces de participación social en el análisis y toma de decisiones. Y en cuarto lugar, ordenamientos y reglas jurídicos, acordadas internacionalmente, para normar el proceso de innovación, desarrollo y evaluación, así como la promulgación de leyes específicas para deslindar responsabilidades civiles y penales imputables a los agentes del desarrollo nanotecnológico. A partir de una nueva experiencia de evaluación social de las nanotecnociencias, es factible extender este modelo a otros sectores de la tecnociencia o del desarrollo tecnológico convencional. De este modo, un intenso debate social y una amplia participación ciudadana conferirá mayor poder a las decisiones tomadas mediante procedimientos de deliberación democrática y plural, y ello podría constituir un verdadero catalizador para poder democratizar sostenidamente los sistemas tecnocientíficos e industriales en el mundo contemporáneo. 
Jorge Enrique Linares Salgado es profesor titular de tiempo completo de la Facultad de Filosofía y Letras de la Universidad Nacional Autónoma de México (UNAM), subdirector del Seminario de Investigación de Ética y Bioética y actualmente coordinador del Posgrado en Filosofía de la Ciencia. Asimismo, pertenece al Sistema Nacional de Investigadores (CONACyTMéxico), nivel II, y es miembro regular de la Academia Mexicana de Ciencias.

Línea de investigación:

Filosofía de la tecnología.

Publicaciones recientes:

(2008) Ética y mundo tecnológico, coeditado por la UNAM y el Fondo de Cultura Económica.

Sitio web: weblinares.wordpress.com

Dirección electrónica: lisjor@unam.mx 